\title{
¿CONDICIONAN LOS RECURSOS NATURALES EL CRECIMIENTO ECONÓMICO?*
}

\author{
Marcela Sánchez Álzate* \\ Recibido: agosto 30 de 2010 \\ Aceptado: octubre 10 de 2011
}

Suponga que en su propio estanque un Lirio de agua está creciendo. La planta de Lirio dobla en tamaño cada día. Si al Lirio le está permitido crecer sin restricción, podría cubrir completamente el estanque en 30 días, asfixiando las otras formas de vida en el agua. Por un largo tiempo la planta parece pequeña y entonces usted decide no preocuparse por cortarla hasta que esta cubre la mitad del estanque. ¿En que día será esto? en el día 29, por supuesto. Usted tiene solo un día para salvar su estanque.

Meadows y otros (1972, p. 29) citado por De Bruyn (2000, p. 1).

\section{RESUMEN}

Este artículo muestra la relación entre recursos naturales y crecimiento económico, mediante una revisión bibliográfica, en la cual se aborda el papel que dichos recursos han tenido en el desarrollo de la teoría económica, su inclusión en los modelos de crecimiento, así como las diferentes perspectivas que sustentan la posibilidad o no de mantener altos niveles de crecimiento económico teniendo en cuenta las limitaciones ambientales. Esta revisión permite concluir que frente a la incidencia que tiene la presencia de recursos naturales para el crecimiento de una economía no existe una única tesis, esto sustentado en que países con abundancia de recursos naturales no han experimentado un rápido y sostenible crecimiento económico, mientras que economías desarrolladas pero limitadas en recursos sí lo han hecho.

\section{PALABRAS CLAVE}

Recursos naturales; crecimiento económico

\section{CLASIFICACIÓN JEL} $\mathrm{O} 13, \mathrm{Q} 32, \mathrm{Q} 56, \mathrm{O} 44$

\section{CONTENIDO}

Introducción; 1. Los recursos naturales en la teoría económica; 2. Los recursos naturales y el crecimiento económico; 3. Diferentes perspectivas a cerca de la relación entre recursos naturales y crecimiento económico; 4. Conclusiones; Bibliografía.

\footnotetext{
Este artículo de revisión bibliográfica es el resultado de una reflexión personal motivada durante el desarrollo de la Maestría en Economía, Facultad de Ciencias Económicas, Universidad de Antioquia, Medellín, Colombia, 2010.

** Ingeniera Administradora, Universidad Nacional, Medellín, Colombia. Economista Universidad de Antioquia, Medellín, Colombia. Magíster en Economía, Universidad de Antioquia, Medellín, Colombia. Docente Universidad de Medellín y Universidad Autónoma Latinoamericana, Medellín, Colombia. Consultora Fundación Grupo HTM, Medellín, Colombia. Dirección postal: carrera 80A N³2D 02 Medellín, Colombia. Teléfono móvil: 3122175218. Correo electrónico: msanchez@grupohtm.org.
} 


\section{DO NATURAL RESOURCES CONDITION ECONOMIC GROWTH?}

\section{ABSTRACT}

This article explains the relation between natural resources and economic growth using a bibliographical review, in which the roll that these resources have played in the economic theory development are analyzed together with their inclusion in growth models as well as the different perspectives that explain the possibility or impossibility of maintaining high economic growth levels taking into account the environmental limitations. This review leads to the conclusion that there is not just one correct thesis for the incidence that natural resources have over economic growth, based on the idea that countries with abundant natural resources have not experimented a fast and sustainable economic growth, while developed economies that have limited natural resources have.

\section{KEY WORDS}

Natural resources; economic growth

\section{JEL CLASSIFICATION}

O13, Q32, Q56, O44

\section{CONTENT}

Introduction; 1. Natural resources in economic theory; 2. Natural resources and economic growth; 3. Different perspectives on the relation between natural resources and economic growth; 4. Conclusions; Bibliography

\section{CONDICIONAM OS RECURSOS NATURAIS O CRESCIMENTO ECONÔMICO?}

\section{RESUMO}

Este artigo mostra a relação entre os recursos naturais e crescimento econômico, mediante a revisão bibliográfica, na qual aborda-se o rol que estes recursos tem desempenhado no desenvolvimento da teoria econômica, sua inclusão nos modelos de crescimento, assim como as diferentes perspectivas que sustentam a possibilidade ou impossibilidade de manter as limitações ambientais. Esta revisão permite concluir que frente à incidência que tem a presencia de recursos naturais para o crescimento de uma economia não existe uma única tese, este sustentado na ideia que os países com abundancia de recursos naturais não tem experimentado um crescimento econômico rápido e sustentável, diferente as econômicas desenvolvidas que apresentam limitações nestes recursos que de fato tem alcançado um alto desenvolvimento.

\section{PALAVRAS-CHAVE}

Recursos naturais; crescimento econômico

\section{CLASSIFICAÇÃO JEL}

O13, Q32, O56, O44

\section{CONTEÚDO}

Introdução; 1. Os recursos naturais na teoria económica; 2. Os recursos naturais e o crescimento económico; 3. Diferentes perspectivas sobre a relação entre os recursos naturais e o crescimento econômico; 4. Conclusões; Bibliografia. 


\section{INTRODUCCIÓN}

¿La abundancia de recursos naturales puede constituirse en un factor decisivo para los procesos de crecimiento económico? o por el contrario cel crecimiento económico se encuentra en conflicto con el medio ambiente? Estos interrogantes han sido planteados y trabajados con mayor refinamiento después de la Segunda Guerra Mundial; sin embargo, no han encontrado una única y acertada respuesta; esto sustentado en que economías ricas en recursos naturales como Nigeria, México o Venezuela no han experimentado un rápido y sostenible crecimiento económico, mientras que economías desarrolladas pero limitadas en recursos naturales, como Japón y Holanda, sí lo han logrado soportadas en su apertura al comercio internacional y su infraestructura de capital.

Las diferencias presentadas entre países frente a sus niveles de crecimiento, en relación con la ausencia o presencia de recursos naturales, de acuerdo con sus condiciones bióticas y abióticas, motivan el desarrollo de este texto de revisión bibliográfica, en el que se aborda la relación existente entre recursos naturales y crecimiento económico, e incluye la revisión del papel que dichos recursos han tenido en la teoría económica y en particular en la teoría del crecimiento, así como las diferentes perspectivas sobre el condicionamiento que ejerce la presencia de los mismos para el crecimiento de una economía.

Las perspectivas incluidas en el texto analizan la relación entre recursos y crecimiento bajo dos enfoques, a saber: el primero de ellos los considera "bendiciones" para el crecimiento, demostrando con evidencias empíricas, su correlación positiva, la cual soportan en la generación de una alta renta económica que permite financiar las fases del desarrollo, y generar materias primas que actúan como suministro para la producción, razón por la cual un aumento en la riqueza del subsuelo genera un efecto directo en el crecimiento. El segundo enfoque, por su parte, los considera "maldiciones", relacionando la abundancia de este tipo de recursos con una distribución desigual de los ingresos, regímenes autoritarios y conflictos civiles, así como con una alta probabilidad de enfrentar la llamada "enfermedad holandesa", dada la volatilidad que experimenta su precio y la incertidumbre asociada a los ciclos de auge y caída.

Una vez revisada la bibliografía, el artículo concluye que no existe una única tesis frente a la relación que ejercen los recursos naturales sobre el crecimiento de las economías, cuestionando para algunos casos la presencia de una correlación positiva entre ellos y poniendo de manifiesto que las economías con abundancia de recursos no siempre mantienen ventaja sobre aquellas con recursos limitados.

El documento se desarrolla en tres apartados: el primero muestra la evolución del papel de los recursos naturales en la teoría económica desde el pensamiento de los clásicos con Malthus (1798) y Ricardo (1817); el segundo, presenta un modelo de crecimiento económico elaborado por William Nordhaus (1992), conocido como "Lethal II Model", el cual según Romer (2006), trabaja una economía cerrada, caracterizada por recursos naturales y tierra, bajo una función de producción del tipo CobbDouglas, que permite demostrar que en el largo plazo una oferta fija de recursos naturales conduce a una caída sostenida del nivel de renta; el apartado final, por su parte, presenta las diferentes perspectivas a través de las cuales se aborda la relación existente entre recursos naturales y crecimiento económico.

\section{LOS RECURSOS NATURALES EN LA TEORÍA ECONÓMICA}

La teoría económica ha considerado la tierra como un recurso determinante para las posibilidades de crecimiento de largo plazo de una economía, no obstante, dado su carácter exógeno, con disponibilidad limitada y rendimientos marginales decrecientes, se convierte en un factor limitante al 
crecimiento, cuyo uso terminaría por reducir el nivel de producción.

Dentro del análisis clásico, se pueden destacar las posiciones de Thomas Malthus (1798) cuya preocupación se manifestó en el crecimiento aritmético de la producción y el crecimiento geométrico de la población, situación determinada por la existencia de una tierra de cultivo limitada y unos rendimientos decrecientes del factor trabajo en la agricultura; según Pearce y Turner (1995), la conclusión de Malthus (1798) giraba en torno a que la producción agrícola sería insuficiente para cubrir el crecimiento de la población; David Ricardo (1817), citado por Pearce y Turner (1995), por su parte, incluyó el tema de la tierra en su análisis económico centrado en los distintos niveles de fertilidad de la misma, en el que concluye que primero se debía cultivar la tierra de mejor calidad, y al tiempo que crecía la población se debían poner en cultivo tierras de peor calidad, lo cual implicaba una menor producción; sin embargo, aun con dichas limitaciones la economía en el largo plazo llegaría al estado estacionario. Por último, de acuerdo con Pearce y Turner (1995, p. 33), John Stuart Mill (1862), analizó las limitaciones que la disponibilidad de tierra puede imponer a los límites del crecimiento, y concluye al decir que los avances tecnológicos pueden contrarrestar los rendimientos decrecientes de la misma.

Naredo (1987) expone que los fisiócratas durante el siglo XVIII, consideraron la naturaleza como única fuente de riqueza, es decir, su enfoque partía de la idea de que toda la riqueza provenía de la tierra y creían que la agricultura era la única rama que generaba producto neto; por tanto, al provenir de la tierra el único excedente, hacia ella debía dirigirse el Estado para obtener fondos, mediante la orientación de la gestión de la economía monetaria a unos principios acordes con las leyes del mundo físico y natural.

De acuerdo con Pearce y Turner (1995, p. 36), la aparición de la teoría neoclásica y con ello la inclusión del sistema de precios y el análisis mar- ginal pusieron de manifiesto la carencia de precios para los bienes y servicios ambientales, razón por la cual se hizo necesaria una nueva concepción de los recursos naturales, que permitiera su incorporación al mercado y un movimiento hacia el uso de bienes menos intensivos en este tipo de recursos, situación que derivó en el desarrollo de figuras como los derechos de propiedad y la internalización de las externalidades, dado que hasta ese momento, los productos proporcionados por el medioambiente eran considerados "gratuitos" y de libre acceso.

Surgen entonces subdisciplinas de la economía tradicional como la economía ambiental, la economía de los recursos naturales, la económica agrícola y la más reciente, la economía ecológica, las cuales desde sus diferentes perspectivas incluyen el tema de los recursos naturales en la teoría económica, establecen límites sobre sus derechos y generan pautas sobre su gestión.

Como parte final de este apartado, es importante resaltar "El Club de Roma", conformado de acuerdo con Meadows (1972), citado por De Bruyn (2000), como una reunión de investigadores y académicos del Instituto Tecnológico de Massachussets, cuya misión era la realización de un estudio sobre las tendencias y los problemas económicos que amenazaban a la sociedad global.

Este estudio puso de manifiesto, por primera vez, la importancia del medioambiente y su esencial relación con la población y la energía; sin embargo, las perspectivas planteadas en él fueron negativas, según Meadows (1972), citado por De Bruyn (2000, p. 25), "si se mantienen las tendencias actuales de crecimiento de la población mundial, industrialización, contaminación ambiental, producción de alimentos y agotamiento de los recursos, este planeta alcanzará los límites de su crecimiento en el curso de los próximos cien años". El resultado más probable, de acuerdo con Meadows (1972), citado por De Bruyn (2000, p. 25), sería un súbito e incontrolable descenso tanto de la población como 
de la capacidad industrial, acompañado de tasas de contaminación muy altas.

Lo anterior denota que la preocupación por la inclusión de la tierra, la naturaleza y en general los recursos naturales se ha presentado a lo largo de la evolución de la teoría económica; sin embargo, estos desarrollos no permiten concluir acerca de la relación existente entre ellos y el crecimiento económico; de manera que se generan procesos posteriores que dan lugar a la modelación de esta relación y a la construcción de evidencias empíricas que ampliarían las perspectivas al respecto.

El siguiente apartado, en el cual se presenta un modelo de crecimiento con recursos naturales como variable dependiente, enriquece la revisión, y permite demostrar que en el largo plazo una oferta fija de recursos naturales conduce a una caída sostenida del nivel de renta

\section{LOS RECURSOS NATURALES Y EL CRECIMIENTO ECONÓMICO}

La inclusión de los recursos naturales en el proceso de desarrollo de la teoría económica permite suponer su importancia para las posibilidades de crecimiento de largo plazo, de la misma manera que los desarrollos que en el ámbito macroeconómico involucran al medioambiente, entre los que se encuentran la curva de Kuznets con la cual se relaciona el ingreso per cápita con la degradación ambiental y el modelo de Nordhaus, el cual incluye el efecto de las emisiones de $\mathrm{CO} 2$ sobre el crecimiento económico.

Sin embargo, los modelos de crecimiento económico no han incluido los recursos naturales y otros aspectos relacionados con el medioambiente como variables determinantes de crecimiento; es posible que esto sea debido a la presunción de rendimientos marginales decrecientes. No obstante, William Nordhaus (1992), hizo una primera aproximación a ello. El modelo de Nordhaus (1992) caracteriza una economía cerrada a través de una función de producción del tipo Cobb-Douglas la cual incluye los recursos naturales y la tierra como variables determinantes del crecimiento. De acuerdo con Romer (2006, p. 39), el modelo de Nordhaus (1992) permite concluir que la presencia de una oferta fija de tierra, una dotación de otros recursos naturales escasa y una producción en continuo crecimiento pueden generar un nivel de contaminación y de destrucción del medioambiente que ponga fin al proceso de crecimiento.

\section{El modelo}

Función de Producción de tipo Cobb-Douglas (Romer, 2006, p. 40):

$$
\mathrm{Y}(t)=\mathrm{K}(t)^{\alpha}{ }_{\mathrm{R}(t)}^{\beta}{ }_{\mathrm{T}(t)} \gamma_{[\mathrm{A}(t) \mathrm{L}(t)]^{1}-\alpha-\beta-\gamma}
$$

Donde:

$$
\begin{aligned}
& \alpha, \beta, \gamma>0 \\
& \alpha+\beta+\gamma<1
\end{aligned}
$$

R: Recursos naturales empleados en el proceso productivo

T: Superficie de Tierra

Y: Producción

K: Capital

L: Trabajo

A: Tecnología o eficacia del trabajo

Tal como lo presenta Romer (2006) y dado que se mantienen los mismos supuestos establecidos en el modelo de crecimiento de Solow (1956), también conocido como modelo Solow-Swan, desarrollado por Solow (1956) y Swan (1956), en cuanto al capital, el trabajo y la tecnología, se tiene que:

$$
\begin{aligned}
& \mathrm{K}(t)=s \mathrm{Y}(t) \mathrm{R}-\delta \mathrm{K}(t) \\
& \mathrm{L}(t)=n \mathrm{~L}(t) \mathrm{L}(t)=n \mathrm{~L}(t) \\
& \mathrm{A}(t)=g \mathrm{~A}(t) \mathrm{A}(t)=g \mathrm{~A}(t)
\end{aligned}
$$

Los nuevos supuestos del modelo se refieren a los recursos naturales y la tierra, esto es: como la superficie de tierra es fija, la cantidad que se emplea 
en el proceso productivo no puede variar en el largo plazo, por ello se supone que:

$$
\mathrm{T}(\mathrm{t})=0
$$

Del mismo modo, el hecho de que la dotación de recursos naturales sea fija y que todos los recursos se empleen en el proceso productivo implica que sus cantidades deben disminuir en algún momento, por tal razón:

$$
R(t)=-6 R(t) \text { con } 6>0
$$

Dados estos supuestos, la pregunta es: ¿existirá o no un estado estacionario? y en caso de existir ¿cuál será la tasa de crecimiento de dichas variables?

Por definición las tasas de crecimiento de A, $\mathrm{L}, \mathrm{R}$ y $\mathrm{T}$ son constantes y solo se requiere para que exista un estado estacionario que K y Y crezcan a una tasa constante e igual; en el momento en que esto ocurra se habrá llegado al estado estacionario.

Al tomar logaritmo natural a ambos lados de la ecuación (1) se tiene que:

$$
\begin{aligned}
\operatorname{Ln} Y(t)= & \alpha \operatorname{Lnk}(t)+\beta \operatorname{LnR}(t)+\gamma \operatorname{LnT}(t) \\
& +(1-\alpha-\beta-\gamma)[\operatorname{Ln} A(t)+\operatorname{Ln} L(t)]
\end{aligned}
$$

A continuación, al derivar (7) con respecto al tiempo se obtienen las tasas de crecimiento del modelo, y se parte del hecho de que la derivada con respecto al tiempo del logaritmo de una variable es igual a la tasa de crecimiento de dicha variable.

$$
\begin{aligned}
g_{Y(t)}= & \alpha g_{K(t)}+\beta g_{R(t)}+\gamma g_{T(t)} \\
& +(1-\alpha-\beta-\gamma)\left[g_{A(t)}+g_{L(t)}\right]
\end{aligned}
$$

Donde:

$g_{\mathrm{Y}(t)}:$ Tasa de crecimiento del producto

Dado que las tasas de crecimiento de las variables $\mathrm{R}, \mathrm{T}$, A y $\mathrm{L}$ son en el mismo orden $-\mathrm{b}, \mathrm{0}$, g y $n$, se puede reescribir (8) como:

$$
g_{Y}=\frac{(1-\alpha-\beta-\gamma)(n+g)-\beta b}{1-\alpha}
$$

Lo cual implica que la tasa de crecimiento de la producción por trabajador en el estado estacionario es:

$$
\begin{aligned}
& g_{\frac{Y}{L}}=g_{Y}-g_{L} \\
& g_{\frac{Y}{L}}=\frac{(1-\alpha-\beta-\gamma) g-\beta b-(\beta+\gamma) n}{1-\alpha}
\end{aligned}
$$

Esto indica que el crecimiento del producto por trabajador puede ser positivo o negativo, y depende del comportamiento de la tierra y los recursos naturales, es decir, la escasez de estos puede hacer que la producción por trabajador disminuya, por tanto, las cantidades decrecientes de recursos y tierra limitan el crecimiento económico, pero el progreso técnico por su parte, lo estimula. Así, si el estímulo generado por el progreso técnico es más fuerte que las restricciones impuestas por los recursos naturales, es posible entonces que la producción por trabajador aumente en forma sostenida. Dicha situación, puede haberse presentado durante los últimos años, en los cuales las ventajas del progreso técnico han compensado los inconvenientes derivados de las escasez de recursos naturales; sin embargo, el alcance de esta restricción podría llegar a ser bastante importante como para que una pequeña desaceleración del progreso técnico conduzca a un crecimiento negativo de la renta por trabajador, según lo expuesto por Romer (2006, p. 42).

Es importante aclarar que dado que el modelo utiliza una función de producción del tipo CobbDouglas, una variación porcentual del nivel de progreso técnico (A) genera variación idéntica del nivel de producción, independiente de cuán elevado sea A en relación con los recursos naturales empleados en el proceso productivo (R) y con la superficie de tierra (T); sin embargo, Romer (2006, p. 43) argumenta que si se utilizara otro tipo de función, por ejemplo, una que se asemeje más a la hipótesis Leontief (1946), los factores cada vez más escasos cobrarían mayor importancia para el modelo, dado que la elasticidad de sustitución entre ellos será menor que 1 y esto generará que la participación en el producto de aquellos factores, cuya dotación es decreciente, aumente en el tiempo. 
No obstante, el desarrollo de este modelo representativo enfatiza la idea de que en el largo plazo una oferta fija de recursos naturales conduce a una caída sostenida del nivel de renta y, por tanto, la economía debe responder a esta situación dirigiéndose hacia procedimientos productivos que empleen los recursos de forma menos intensiva, o a la aplicación de instrumentos económicos que garanticen el crecimiento, a su vez que se limita el agotamiento de los recursos.

Es allí donde cobra importancia el papel de las instituciones a través de la formulación de mecanismos y políticas económicas ambientales que propendan por alivianar el deterioro ambiental ocasionado por altos niveles de crecimiento. En palabras de Acemoglu, Jonson y Robinson (2004, p. 2): "las instituciones importan en el crecimiento pues ellas condicionan la inversión en capital físico y humano, en tecnología y en la organización de la producción".

Si bien este modelo presenta una primera aproximación a una correlación negativa entre crecimiento económico y escasez de recursos naturales, aparecen en la literatura argumentaciones que propenden por una relación positiva entre estos. En este sentido, el siguiente apartado sintetiza las diferentes percepciones existentes frente a la relación entre recursos naturales y crecimiento económico. Su desarrollo permite mostrar a partir de evidencias empíricas, el amplio desacuerdo que frente a dicha relación se presenta y en consecuencia responder a la pregunta planteada como título del presente artículo.

\section{DIFERENTES PERSPECTIVAS ACERCA DE LA RELACIÓN ENTRE RECURSOS NATURALES Y CRECIMIENTO ECONÓMICO}

El debate sobre recursos naturales y crecimiento económico es reciente, inició a finales de la década de los sesenta y comienzos de la década de los setenta una vez que los reportes del Club de Roma avisaban sobre los efectos del crecimiento económico exponencial frente a daños ambientales tales como el incremento de la contaminación, la sobreexplotación de recursos renovables y el agotamiento de recursos no renovables, según lo argumenta De Bruyn (2000).

No obstante, a comienzos de la década de los setenta aparecen dos diferentes percepciones frente a la relación entre crecimiento y recursos naturales; la primera de ellas sustentaba la imposibilidad de mantener niveles de crecimiento sostenibles en el ámbito ambiental, mientras que la segunda, por el contrario, sugirió que el crecimiento económico podría ser benéfico para la calidad del medioambiente y que la vía para mejorar las condiciones ambientales se soportaba en altos niveles de crecimiento; solo así se lograrían mayores inversiones en investigación y desarrollo (I+D) y la generación de fondos para financiar políticas ambientales. Lo anterior, de acuerdo con Field y Field (2003, p. 13), se basa en el hecho de que la contaminación aumenta durante las primeras fases de desarrollo de los países y luego comienza a disminuir a medida que se obtienen los recursos adecuados para hacer frente a los problemas que ella misma genera.

De Bruyn (2000, p. 5) expone que estas diferentes percepciones acerca de la relación entre crecimiento económico y recursos naturales, al adoptar como proxy la calidad del medioambiente, permitieron clasificar los diferentes enfoques que ha tenido el debate entre crecimiento y recursos, de la siguiente manera:

1. La posición del defensor radical: según De Bruyn (2000) esta posición postula una relación positiva entre crecimiento económico y calidad ambiental, dado que altos niveles de crecimiento producen fuertes innovaciones tecnológicas y cambios en los estilos de vida que pueden mejorar el medioambiente. Las recomendaciones de política son: incentivar el crecimiento y remover las barreras que obstaculizan la llegada de nuevas tecnologías y la efectiva protección al medioambiente. Se soporta en la utilización de 
mecanismos de mercado para aliviar los problemas ambientales.

2. La posición del defensor condicional: al igual que la anterior, asume una correlación positiva entre crecimiento y medioambiente; sin embargo, considera que mayores niveles de producción pueden tener efectos adversos para la calidad ambiental, pero, a su vez, esta mayor producción, incrementaría los fondos con los cuales pueden ser financiadas las políticas ambientales. Por tanto, acorde con World Bank (1992) y Grossman y Krueger (1994), citados por De Bruyn (2000, p. 5), la mejor vía para mantener la calidad del ambiente es simular altos niveles de crecimiento e implementar al mismo tiempo políticas sanas en pro de la conservación ambiental.

3. La posición del antagonista débil: postula que altos niveles de crecimiento causan deterioro al medioambiente; sin embargo, este detrimento podría ser mitigado por políticas ambientales, las cuales resultan ser menos efectivas que el mismo crecimiento económico, por tanto, la única solución existente consiste en reducir los niveles de crecimiento de ciertos sectores de la economía, con el fin de mejorar la calidad ambiental, acorde con Arrow (1995), citado por De Bruyn (2000, p. 6).

4. La posición del antagonista fuerte: considera que el crecimiento económico de largo plazo va siempre en contra de la calidad ambiental, por ello, políticas que mitiguen el deterioro ambiental pueden tener efectos temporales pero no mejoras sustanciales a la calidad ambiental. Según Meadows (1972), citado por De Bruyn (2000, p. 6), la recomendación de política es reducir el crecimiento económico en general.

Dadas las consideraciones anteriores, es posible afirmar que la pregunta: ¿el crecimiento económico se encuentra en conflicto con el medioambiente?, no ha encontrado una única y acertada respuesta y la evidencia generada en el intento por resolverla pone de manifiesto que no en todos los casos las economías ricas en recursos tienen ven- taja sobre aquellas con recursos limitados. Según Sachs y Warner (2001, p. 2), economías como la de Nigeria, México o Venezuela que con abundancia en recursos naturales no han experimentado rápido y sostenible crecimiento económico, o aquellas como la japonesa, que con escasez de recursos naturales ha experimentado altos niveles de crecimiento, son ejemplos de esta condición.

Dado lo anterior, se pueden diferenciar dos enfoques: uno que considera los recursos naturales como "bendiciones" para el crecimiento y sustenta una correlación positiva entre ellos, y otro que los califica como "maldiciones" que supone una relación negativa entre los mismos.

La corriente que considera que los recursos naturales actúan como "bendiciones" que soportan y aceleran el crecimiento económico argumenta que estos proporcionan una ventaja comparativa significativa con relación a otros sectores de la economía, en virtud de la generación de renta económica; dado que la abundancia de recursos en un territorio permite financiar las fases del desarrollo, y generar materias primas que actúan como suministro para la producción, y renta económica, una vez son exportados a los mercados del mundo; de acuerdo con Mackintosh (1964), citado por Gunton (2003). Este hecho justificaría la transformación industrial de países como Gran Bretaña, Alemania, Estados Unidos y los más recientes, Noruega e Islandia quienes lograron desarrollar un sector primario de alto nivel capaz de generar altas rentas.

En particular, los recursos minerales, pueden llegar a tener una asociación directa con el crecimiento real del PIB, debido a que un aumento en la riqueza del subsuelo generará un efecto directo en el crecimiento. Noruega es un ejemplo de esta situación a través de sus reservas de petróleo; así lo demostró Brunnschweiler (2008) a través de un trabajo empírico para diferentes países en todos los continentes, durante el período comprendido entre 1970 y 2000. 
En este mismo sentido, es interesante rescatar el trabajo de Gallup, Sachs y Mellinger (1999, p. 11), en el cual se pone de manifiesto que la proximidad y pertenencia a recursos naturales tales como agua, minerales, piedras preciosas y depósitos de hidrocarburos son variables relevantes para el desarrollo económico, aunado a condiciones geográficas como la extensión de tierra en los trópicos geográficos, la proporción de la población dentro de los 100 Kms de la línea de costa o de un río navegable y la distancia y acceso al mar. Estas condiciones, además, han motivado a que por siglos la población se ubique en regiones ricas en recursos naturales, como es el caso de gran parte del continente americano, Australia y del Medio Este, dado que los recursos proveen una base para el desarrollo, tal como lo expone World Bank (2005, p. 2).

Esta visión que considera los recursos naturales "bendiciones" da, a su vez, un papel preponderante a las instituciones en su rol frente al crecimiento de una economía. Si bien no hay pruebas de que la abundancia de recursos afecte de forma negativa a la calidad institucional, la eficacia del gobierno y el incremento en los índices de calidad institucional han tenido un efecto positivo en el crecimiento y han asumido una relación de causalidad directa entre ellos, argumenta Brunnschweiler (2008).

Sin embargo, existen otras consideraciones sobre el hecho de que la cercanía y abundancia a recursos naturales actúa como una "maldición" para las economías, razón por la cual países ricos en este tipo de recursos presentan una distribución desigual de sus ingresos, regímenes autoritarios y una alta probabilidad de enfrentar conflictos armados: la probabilidad de enfrentar una guerra civil en un país con recursos naturales limitados es del $5 \%$, mientras que en un país rico en recursos naturales, la probabilidad crece al $23 \%$, este es el caso de las economías africanas, según lo indican Collier y Venables (2008) y Collier y Goderis (2008).

Sachs y Warner (1995 y 2001), por su parte, encontraron asociación negativa entre el crecimiento económico y la abundancia de recursos naturales, mediante un trabajo empírico que utilizó tasas de crecimiento del PIB para países con abundancia de recursos entre los que se encuentran Nigeria, México, Venezuela, Costa de Marfil, Arabia Saudí y Kuwait, durante el periodo comprendido entre 1970 y 1990. Mediante el uso de regresiones que controlaban las variables geografía y clima, mostraron que existía evidencia de que estas variables explican la denominada maldición de los recursos naturales, a la que atribuyeron los ciclos de estancamiento presentados por dichas economías durante las décadas analizadas. Este resultado se puede considerar opuesto al planteado por Gallup y otros (1999), en el cual, cómo se mostró con antelación, las condiciones geográficas soportan el desarrollo económico.

Sachs y Warner (1995 y 2001) sustentaron, además, que los efectos de la abundancia de recursos naturales en un país, dependen de las rentas económicas de dichos recursos y, por tanto, existe diferencia en las tasas de crecimiento de países con alta presencia de recursos minerales que mantienen altas rentas, comparadas con aquellos con abundancia de recursos agrícolas, cuyas rentas son menores; tal es el caso de Costa de Marfil o Mauritania. Por último, Sachs y Warner (1995 y 2001) impulsaron la idea de que muchos países con abundancia de recursos tienden a tener altos precios y como consecuencia se estancan a la espera de un crecimiento impulsado solo por las exportaciones.

Lo anterior está asociado al hecho de que la presencia de recursos de alto valor, tales como hidrocarburos y piedras preciosas, condiciona a un país a la llamada "enfermedad holandesa", donde los altos niveles de exportación de dicho recurso en abundancia provocan un flujo monetario hacia el país y, por tanto, una apreciación de los tipos de cambio. De esta forma, resulta cada vez más sencillo y barato para el país importar productos transables, mientras que para la industria nacional es cada vez más difícil exportar su producción, dado 
que se genera alta inversión interna en bienes no transables para la economía.

Otro efecto negativo de la abundancia de recursos naturales, de acuerdo con The World Bank (2005, p. 2), está asociado a la volatilidad que experimenta el precio de los bienes naturales, dada la incertidumbre asociada a los ciclos de auge y caída. Esta situación se presentó, por ejemplo, en 1970 cuando los elevados precios de los hidrocarburos llevaron a los países con abundancia en ellos a solicitar préstamos que más adelante les generaron grandes deudas y poca capacidad de servicio a las mismas, una vez que en 1980 el precio descendió; tal es el caso de economías como las de Bolivia, Venezuela y Nigeria.

Según esta corriente, la maldición de los recursos naturales sería un efecto indirecto de la abundancia de los mismos y un determinante significativo de bajos niveles de crecimiento, debido a la disminución en inversión, escolaridad, apertura y gasto en investigación y desarrollo (I+D), así como de un incremento en la corrupción, situación que se ha manifestado en los países que conforman el denominado cartel del petróleo; economías ricas en recursos pero que tienden a invertir poco en educación e infraestructura están en constante búsqueda de altas rentas y, a su vez, mantienen niveles elevados de corrupción, por lo cual no logran diversificar sus economías ni limitan la ineficacia del gobierno, según lo argumentan Papyrakis y Gerlagh (2007, p. 19).

Un enfoque adicional asociado a la llamada maldición de los recursos naturales, según Dasgupta (1990) y Chichilnisky (1994), citados por Arrow y otros (2003, p. 25), está determinado por el hecho de que los países con abundancia de recursos naturales pueden exportarlos a países con bajo stock y subsidiar así su consumo. No obstante, tales subsidios ocultos ayudarían a promover tasas positivas de crecimiento per cápita en los países importadores, mientras que en los países exportadores solo se concentrarían menores tasas de crecimiento y un alto nivel de degradación ambiental, poniendo en peligro su bienestar.

Esta pérdida de riqueza de las naciones con abundancia de recursos naturales está relacionada con el bajo costo de utilización de los mismos. En este sentido, los recursos naturales a excepción del petróleo, son valorados por debajo de su costo social; este hecho, según Arrow y otros (2003), se encuentra asociado a una errada definición de los derechos de propiedad y a una incapacidad del mercado para incorporar las externalidades derivadas del uso de los recursos naturales, tales como daños diversos generados por combustibles fósiles o la pérdida de servicios ambientales como control de inundaciones y regulación hídrica.

De manera adicional, la relación negativa entre recursos y crecimiento, según Sachs y Warner (2001), podría verse afectada por la poca eficiencia burocrática o ineficiencia del gobierno; sin embargo, la denominada "maldición" de los recursos naturales no es un problema de los países con instituciones débiles, pero este hecho se convierte en una amenaza potencial tanto para las economías en desarrollo como para las desarrolladas, y su manejo puede revertir el curso de la "maldición" de los recursos, exponen Papyrakis y Gerlagh (2007, p. 19); Nuevo México y Texas, en Estados Unidos, con alta inversión en investigación y desarrollo (I+D) durante el período comprendido entre 1986 y 2000, son ejemplos de que las políticas económicas acertadas y la planificación prudente revierten la maldición de los recursos y en este sentido regiones con abundancia de recursos pueden tener una ventaja comparativa para el desarrollo, indican Papyrakis y Gerlagh (2007, p. 18).

Estas consideraciones anteriores, en alguna medida, explicarían por qué los africanos, quienes conservan alrededor del 50\% más de tierra por persona que el promedio mundial, sustentan menos capital físico y humano por persona, tal y como lo indican Sachs y Warner (1995). 


\section{CONCLUSIONES}

Si bien la sola presencia de recursos naturales en un territorio ofrece una ventaja para su crecimiento económico, encontrar una respuesta única y acertada al cuestionamiento sobre la relación entre recursos naturales y crecimiento económico resulta una tarea compleja. La abundancia de recursos de este tipo ha influido en el crecimiento y la diversificación de países como Australia, Canadá, Finlandia y Noruega y ha proveído una base para el desarrollo de sus economías, mientras que la presencia de estos no ha significado incremento en los niveles de renta de las economías africanas, por el contrario, ha limitado sus proyecciones de crecimiento.

Aunque la teoría económica ha incluido el tema de los recursos naturales desde sus análisis iniciales, el hecho de que este tipo de recursos presente rendimientos marginales decrecientes hace compleja su inclusión en los modelos de crecimiento; no obstante, se han hecho algunas aproximaciones al tema, pero la evidencia empírica puede llegar a contrarrestar los resultados de las mismas.

Mientras que la abundancia de recursos naturales proporciona una ventaja comparativa significativa con relación a otros sectores de la economía, en virtud de la generación de renta económica, se encuentra una relación negativa entre crecimiento y recursos que podría verse afectada por variables como bajas tasa de inversión y presencia de instituciones débiles.

Permanece entonces el interrogante sobre si la degradación del medioambiente y de los recursos naturales, puede ser ocasionada por un excesivo desarrollo económico o, por el contrario, por un desarrollo económico insuficiente, con pocas políticas y mecanismos que permitan reducir los efectos del daño ambiental, sin necesidad de reducir los niveles de crecimiento.

\section{BIBLIOGRAFÍA}

Acemoglu, Daron; Jonson, Simon y Robinson, James (2004). Institutions as the Fundamental Cause of Long-Run Growth. En: Handbook of Economic Growth, Vol. 1A, p. 354-464.

Arrow, K. (1995). Economic Growth, Carrying Capacity and the Environment. En: Science, Vol. 268, p. 520-521.

Arrow, Kenneth; Dasgupta, Partha; Goulder, Lawrence; Daily, Gretchen; Ehrlich, Paul; Heal, Geoffrey; Levin, Simon; Mäler, Karl-Göran; Schneider, Stephen; Starrett, David, y Walker, Brian (2003). Are We Consuming Too Much?. En: Journal of Economic Perspectives, Vol.18, No. 3, p.147-172.

Brunnschweiler, Christa N. (2008). Cursing the Blessings? Natural Resource Abundance, Institutions and Economic Growth. En: World Development, Vol. 36, No 3, p. 399-419.

Chichilnisky, G. (1994). North-South Trade and the Global Environment. En: American Economic Review, Vol. 84, No 3, p. 851-874.

Collier, Paul y Venables, Anthony (2008). Managing the Exploitation of Natural Assets: Lessons for Low Income Countries. African Economic Research. Consortium 2008 Annual Conference, Nairobi, 28p.

Collier, Paul y Goderis, Benedikt (2008). Commodity Prices, Growth, and the Natural Resource Curse: Reconciling a Conundrum. Department of Economics, University of Oxford, 45p.

Dasgupta, P. (1990). The Environment as a Commodity. En: Oxford Review of Economic Policy, Vol. 6, No 2, p. 51-67.

De Bruyn, Sander M. (2000). Economic Growth and the Environment: an Empirical Analysis. Kluwer Academic Publisher, The Netherlands, 231p.

Field Barry C. y Field Martha K. (2003). Economía Ambiental. $3^{a}$ ed, Mc Graw Hill, Madrid, 556p.

Gunton, Thomas (2003). Natural Resources and Regional Development: an Assessment of Dependency and Comparative Advantage Paradigms. En: Economic Geography, Vol. 79, No 1, p. 67-94. 
Gallup, John Luke; Sachs, Jeffrey D. y Mellinger, Andrew D. (1999). Geography and Economic Development. United States Agency for International Development. Consulting Assistance for Economic Reform (CAER) II Project, Contract No.PCEC-00-95-00015-00, 59p.

Grossman, G.M.; Krueger, A.B. (1994). Economic Growth and the Environment. En: NBER Working Paper, No 4634.

Leontief, W. (1946). The pure Theory of the Guaranteed Annual Wage Contract. En: Journal of Political Ecoonomy, No 54, p. 76-79.

Mackintosh, W. A. (1964). The economic background of dominion-provincial relations. McClelland and Stewart, Toronto.

Malthus, Thomas Robert (1798). An Essay on the Principle of Population, as It Affects the Future Improvement of Society. J. Jonson, Londres.

Meadows, Donella H.; Meadows, Dennis L.; Randers, Jorgen, y Behrens, William W. (1972). The Limits to Growth. Universe Books, New York.

Mill, John Stuart (1862), Principles of Political Economy. Appleton, New York.

Naredo, José Manuel (1987). La Economía en Evolución. Siglo XXI de España Editores, Madrid, 538p.

Nordhaus, William D. (1992). Lethal Model 2: The Limits to Growth Revisited. En: Brookings Papers on Economic Activity, No 2, p. 1 43.

Pearce, David W. y Turner, Kerry R. (1995). Economía de los Recursos Naturales y del Medio Ambiente. Colegio de Economistas de Madrid. Celeste Ediciones, Madrid, 448p.
Papyrakis, Elissaios y Gerlagh, Reyer (2007). Resource Abundance and Economic Growth in the United States. En: European Economic Review, No 51, p. 1011-1039.

Romer, David (2006). Macroeconomía Avanzada. $3^{a}$ ed, McGraw- Hill Companies, Madrid, 690p.

Ricardo, David (1817). Principles of Political Economy and Taxation. Pelican Books, Londres.

Sachs, Jeffrey D y Warner, Andrew M (1995). Natural Resource Abundance and Economic Growth. NBER Working Paper Series. En: National Bureau of Econonomic Researc, Massachusetts, p. 1-54.

Sachs, Jeffrey y Warner, Andrew (2001). Natural Resources and Economic Development: The Curse of Natural Resources. En: European Economic Review, No 45, p. 827-838.

Solow, Robert M. (1956). A Contribution to the Theory of Economic Growth. En: Quarterly Journal of Economics, No 70, p. 65-94.

Swan, T. W. (1956). Economic Growth and Capital Accumulation. En: Economic Record, No 32, p. 334-361.

World Bank (1992). World Development Report, World Bank.

World Bank (2005). Natural resources: When Blessings Become Curses. Country Note H. Economic growth in the 1990's, Learning from a decade of reform, Washington, p. 308-311. 